\title{
Cumplimiento de derechos infantiles en Chile: evaluación de profesionales de programas públicos
}

\author{
Giselle Lagos Beltrán, Mg. ${ }^{a}$ \\ Universidad de La Frontera, Chile \\ Ricardo Pérez-Luco Arenas, Ph.D. ${ }^{b}$ \\ Universidad de La Frontera, Chile
}

giselle.lagos@ufrontera.cl

\section{Resumen (analítico)}

Ante la escasez de evidencia, el objetivo general de este estudio fue describir y aplicar un instrumento propuesto para medir los derechos infantiles en Chile, desde el ejercicio cotidiano de operadores sociales que trabajan con infancia y adolescencia en centros educacionales, de salud, justicia o de desarrollo psicosocial. La muestra estuvo compuesta por 407 participantes (profesores, psicólogos, entre otros). El instrumento considera los cuatro principios fundamentales de la Convención de Derechos del Niño: no discriminación, interés superior, derecho a la vida, desarrollo, supervivencia y participación. Los resultados preliminares sugieren que existen diferencias estadísticamente significativas en el grado de cumplimiento de los derechos según el ámbito de desempeño. Se discute la consistencia interna del instrumento y su adaptación según ámbito, población atendida y aplicación en población infantil general y específica.

\section{Palabras clave}

Responsabilidad del Estado, derechos del niño, instrumento de medición, trabajador social.

\section{Thesauro}

Tesauro de Ciencias Sociales de la Unesco.

\section{Para citar este artículo}

Lagos, G., \& Pérez-Luco Arenas, R. (2021). Cumplimiento de derechos infantiles en Chile: evaluación de profesionales de programas públicos. Revista Latinoamericana de Ciencias Sociales, Niñez y Juventud, 19(1), 1-19.

https://dx.doi.org/10.11600/rlcsnj.19.1.4224

\section{Historial}

Recibido: 03.06.2020

Aceptado: 26.07 .2020

Publicado: 04.12.2020

\section{Información artículo}

Este artículo es parte de la investigación denominada Evaluación del Cumplimiento de la Convención de los Derechos del Niño según la opinión de operadores sociales en Chile, presentada por la autora para optar al grado de Magíster en Psicología de la Universidad de La Frontera. Investigación realizada entre agosto 2015 y enero 2017. Estudios de posgrado financiados por Conicyt-PCHA/Magíster Nacional/2015 - Folio 22150877. Área: Ciencias Sociales; subárea: Sociología. 


\section{The fulfilment of children's rights in Chile: Evaluation of public program staff}

\section{Abstract (analytical)}

Given the lack of evidence in this area, the general objective of this study was to describe and apply an instrument designed to measure children's rights in Chile based on the day to day practices of social operators who work with children and adolescents, in educational, health, justice and psychosocial development centers. The sample consisted of 407 participants (including teachers and psychologists). The instrument takes into account the four fundamental principles of the Convention on the Rights of the Child: non-discrimination; the best interest of the child; the right to life, development and survival; and the right to be heard. The preliminary results suggest that there are statisticaIly significant differences in the degree of fulfillment of rights in relation to each fundamental princi-

ple. The internal consistency of the instrument, its adaptation to measure the fulfillment of each principle and the population that receive assistance are discussed, as well as application of the instrument with general and specific child populations.

Keywords

State responsibility, children's rights, evaluation instruments, social workers.

\section{Conformidade com os direitos da criança no Chile: Avaliação de profissionais de programas públicos}

Resumo (analítico)

Dada a falta de evidências, o objetivo geral deste estudo foi descrever e aplicar um instrumento proposto para medir os direitos da criança no Chile, a partir da prática diária de operadores sociais que trabalham com crianças e adolescentes, em centros educacionais, de saúde, justiça ou desenvolvimento psicossocial. A amostra foi composta por 407 participantes (professores, psicólogos, entre outros). O instrumento considera os quatro princípios fundamentais da Convenção sobre os Direitos da Criança: Não discriminação; Interesse superior; Direito à vida, à sobrevivência e ao desenvolvimento; Participação. Os resultados preliminares sugerem que existem diferenças estatisticamente significativas no grau de cumprimento dos direitos de acordo com o âmbito de desempenho. Além disso, o trabalho discute a consistência interna do instrumento e sua adaptação segundo o campo, população atendida e aplicação em população infantil geral e específica.

\section{Palavras-chave}

Responsabilidade do Estado, os direitos das criança, instrumento de medição, assistente social.

\section{Información autores}

[a] Magíster en Psicología, Universidad de La Frontera. H5: 0. (iD) 0000-0003-3144-8548. Correo electrónico: giselle.lagos@ufrontera.cl

[b] Psicólogo, Universidad de La Frontera. Magíster en Ciencias Sociales y Políticas, Flacso, México. Doctor en Psicología, Universidad Pontificia de Salamanca. Profesor e Investigador Universidad de La Frontera, Chile. H5: 9 iD 0000-0002-7051-5005. Correo electrónico: ricardo.perez-luco@ufrontera.cl 


\section{Introducción}

$\mathbf{E}_{\text {particularmente en Chile, resuenan las demandas y necesidades latentes de }}$ los niños, niñas y adolescentes (NNA) que históricamente han sido vistos como objetos de protección y con un rol social difuso (siglos XVIII y XIX) (Amador, 202o; Contreras-Taibo et al., 2018; Herrera \& Aravena, 2015).

Luego de casi tres décadas de que el país retornara a la democracia y ratificara la Convención Internacional de los Derechos del Niño (Ministerio de Relaciones Exteriores, 1990), se mantiene el desafío de avanzar en la evaluación de programas que atienden a niños, niñas y adolescentes ante la escasez de evidencia científica que se tiene sobre el impacto de las acciones realizadas en el trabajo con la infancia (Muzzio et al., 2008).

En Chile se observa una desarticulación entre lo declarado en la Convención y las prácticas cotidianas (Herrera \& Aravena, 2015; Observatorio para la Confianza, 2019), pues los niños y las niñas son titulares de sus derechos solo a nivel discursivo (Contreras, 2007; Dávila \& Naya, 2006; Sola-Morales \& Campos-Garrido, 2019).

La Convención plantea cuatro principios fundamentales que buscan garantizar el bienestar pleno de la infancia considerando primordial el interés superior (Beloff, 1990; Giorgi, 2010; Naciones Unidas, 2013; Save the Children, 2009). Lo anterior, como marco jurídico internacional, ha sido la base para la creación y aplicación de índices e instrumentos de medición desde macrodimensiones o condiciones generales con diferentes experiencias a nivel latinoamericano y mundial (Alkire \& Foster, 2011; Chzhen et al., 2016; De Neubourg et al., 2012; Espinoza-Delgado \& Klasen, 2017; Guio et al., 2012; Klasen, 2018; Langford \& Kirkebø, 2019; Sen, 1979; Thorbecke, 2008).

A su vez, en Chile se encuentran instrumentos y estudios mayoritariamente desde macrodimensiones. Por ejemplo, derechos para medir la pobreza infantil a nivel del espacio del hábitat de vida, el derecho a la alimentación, indicadores de desarrollo humano, desarrollo social y derechos humanos (Antilef, 2015; Cepal \& Unicef, 2012; Espíndola et al., 
2017; Observatorio Social, 2015; Oyanedel et al., 2015; Pautassi \& Royo, 2012). No obstante, se hace urgente avanzar en el desarrollo de mediciones multidimensionales que evalúen las prácticas cotidianas y aporten a un modelo integral para monitorear las acciones realizadas en infancia y adolescencia a partir de cifras oficiales (Fondo de las Naciones Unidas para la Infancia, 2015; Observatorio de la Niñez y Adolescencia, 2017; Tuñón \& González, 2013).

El Comité de Derechos del Niño de Naciones Unidas entregó en 2018 un informe que concluyó que el Estado de Chile es responsable de la vulneración grave y sistemática de los derechos de niños, niñas y adolescentes vulnerables a su cargo durante los últimos 40 años. Además, se reforzó lo indicado en informes previos sobre la ausencia de una ley de protección integral desde el enfoque de derechos. Al mismo tiempo, la falta de voluntad o incompetencia del Estado para tomar medidas eficaces y oportunas pese a contar con variados informes oficiales de los poderes ejecutivo, judicial y legislativo que diagnosticaban la situación del sistema de protección residencial (Comité de los Derechos del Niño, 2018).

Ante la ausencia de una ley integral de garantía de derechos infantiles, Magistris (2013) plantea dos riesgos: el primero que releva el tener el mejor estándar sobre cómo se implementan los derechos infantiles, equiparando «el enfoque de derechos a un discurso meramente tecnocrático, sin cuestionamientos ni problematización respecto de las relaciones de poder en la que se hallan inmersos niños y niñas» (p. 10); el segundo es un riesgo de descontextualización, que promueve derechos universalistas, sin considerar las características sociohistóricas y culturales del entorno local de los niños.

Por otro lado, la Convención exige a los Estados miembros contar con personal competente en las instituciones y servicios vinculados al cuidado de la infancia. Tal personal también es conocido como operadores sociales debido a su desempeño laboral, quienes implementan las leyes y las políticas públicas (Ministerio de Relaciones Exteriores, 1990). En Chile estos son mayoritariamente profesionales, quienes deben cumplir tanto los principios fundamentales de la Convención como las demandas y responsabilidades específicas de la institución o del programa en que están insertos (Alfaro, 2012; Alfaro \& Zambrano, 2009).

En los escasos estudios realizados en Chile sobre la opinión de los operadores sociales (Contreras \& Pérez, 2011; Herrera \& Aravena, 2015; Marina, 1998) llama la atención que predomina una visión de la infancia como objeto de protección, con una incipiente concepción de sujetos titulares de derecho. 
Así mismo, en el estudio de Durán-Strauch (2017) sobre política pública de infancia y adolescencia de Bogotá, se concluyó que existen discursos tensionados sobre la garantía de derechos al analizar lo planteado en la política local y la práctica de instituciones. Por su parte, en la encuesta realizada a médicos en formación como especialistas en pediatría (Zunino et al., 2018), se concluyó que si bien el $97 \%$ conoce las normativas nacionales e internacionales sobre derechos infantiles, el 25\% no las aplica en la resolución de problemas y, además, ha observado vulneración de derechos en el contexto de su formación y práctica clínica.

Para profundizar en el tema, este estudio se propuso como objetivos: a) describir la consistencia interna del instrumento a través del alpha de Cronbach; y b) establecer si existen diferencias en la evaluación por dimensión de acuerdo al ámbito de desempeño del programa o institución de adscripción de los operadores sociales (salud, educación, justicia y desarrollo social).

\section{Método}

\section{Diseño y participantes}

Para dar cumplimiento a los objetivos de la investigación, se utilizó un diseño no experimental, con metodología cuantitativa, mediante muestreo no probabilístico intencionado de autoselección (Eiroá et al., 2008; Hernández et al., 2010), obteniendo una muestra de 407 operadores sociales.

El tamaño muestral se calculó a priori para pruebas $F$ (Anova de una vía) mediante el software G*Power (versión 3.1), considerando, debido a la poca evidencia teórica y empírica existente, un error $\beta=0.95$, un error $\alpha=0.05$ y un tamaño de efecto moderado en su nivel más bajo $(f=0.25)$, lo cual arrojó un tamaño muestral mínimo de 280 casos.

Los criterios de inclusión fueron: a) trabajar o haber trabajado con niños, niñas y adolescentes; b) por al menos un año; c) en instituciones o programas del área de la salud, educación, justicia o desarrollo social. En términos generales, la muestra estuvo compuesta por $98 \%$ de técnicos o profesionales (solo $2 \%$ tenía estudios básicos o secundarios), entre los cuales la mayor participación fue de profesores (31\%), psicólogos (24.8\%), educadores diferenciales (7.4\%) y educadores de párvulos (7.4\%) (tabla 1). 


\section{Tabla 1}

Caracterización de la muestra

\begin{tabular}{|c|c|c|c|c|c|c|c|}
\hline \multirow{2}{*}{$\begin{array}{l}\text { Área de programa } \\
\text { o institución }\end{array}$} & \multicolumn{2}{|c|}{ Sexo } & \multirow{2}{*}{ Edad } & \multirow{2}{*}{$\begin{array}{l}\text { Área de formación } \\
\text { educacional de } \\
\text { origen }\end{array}$} & \multirow{2}{*}{$\begin{array}{c}\text { Años de } \\
\text { experiencia } \\
\text { con NNA }\end{array}$} & \multirow{2}{*}{$\begin{array}{c}\text { Cantidad } \\
\text { de trabajos } \\
\text { con NNA }\end{array}$} & \multirow{2}{*}{$\begin{array}{l}\text { Total } \\
(\mathrm{N})\end{array}$} \\
\hline & $M$ & $F$ & & & & & \\
\hline Salud & 16 & 45 & $\bar{X}=34$ & $\begin{array}{l}67 \% \text { salud } \\
23 \% \text { ciencias sociales }\end{array}$ & $\bar{X}=7,1$ & $\bar{X}=3$ & 61 \\
\hline Educación & 47 & 227 & $\bar{X}=36$ & $\begin{array}{c}70 \% \text { educación } \\
21 \% \text { ciencias sociales }\end{array}$ & $\bar{X}=10$ & $\bar{X}=3.7$ & 274 \\
\hline Justicia & 8 & 43 & $\bar{X}=35$ & $\begin{array}{l}80 \% \text { ciencias sociales } \\
10 \% \text { ciencias jurídicas }\end{array}$ & $\bar{X}=7.3$ & $\bar{X}=3.6$ & 51 \\
\hline Desarrollo social & 6 & 15 & $\bar{X}=35$ & $\begin{array}{l}76 \% \text { ciencias sociales } \\
14 \% \text { educación }\end{array}$ & $\bar{X}=7.2$ & $\bar{X}=3.5$ & 21 \\
\hline Total & $19 \%$ & $81 \%$ & $\bar{X}=35$ & & $\bar{X}=7.9$ & $\bar{X}=3.45$ & 407 \\
\hline
\end{tabular}

\section{Instrumento de medición}

El instrumento usado se construyó previo a la investigación (Lagos, 2017), quedando compuesto por 59 ítems (tabla 2), agrupados en cuatro dimensiones de acuerdo a los principios fundamentales de la Convención de Derechos del Niño (Ministerio de Relaciones Exteriores, 1990) (tabla 3). Posee 5 opciones de respuesta en formato tipo Likert: nunca se cumple $=1$, rara vez $=2$, a veces sí, a veces no $=3$, casi siempre $=4$ y siempre se cumple $=5$. Además, incluye una sexta opción (no aplica), asumiendo la posibilidad que en su experiencia algún operador social no hubiese tenido la oportunidad de observar el cumplimiento del derecho consultado. Tales dimensiones difieren en la cantidad de ítems según la validación de contenido realizada en una etapa de la investigación (Lagos, 2017). Adicionalmente, se incluyó zo preguntas sociodemográficas y laborales. 


\section{Tabla 2}

\section{Escala de medición multidimensional de derechos infantiles (Emmdi)}

\section{Ítem Indique con qué frecuencia ocurren las siguientes afirmaciones en el programa o institución en que usted trabaja con niños, niñas o adolescentes}

\footnotetext{
1 Se respeta a cada niño independiente de su etnia.

2 No existen diferencias en el trato que se da a los niños según su apariencia física.

3 No se hacen distinciones en el trato a los niños según su nacionalidad.

4 Se hacen distinciones en el trato a los niños según su posición socioeconómica.

5 Se hacen distinciones en el trato según el género del niño o adolescente.

6 Se hacen diferencias en el trato a los niños con impedimentos físicos.

7 Mediante la educación, se inculca a los niños el respeto de los derechos humanos.

8 Por medio de la educación, se está inculcando a los niños a respetar su propia identidad cultural.

9 Todo niño tiene acceso a la educación básica, media o superior.

10 Se garantiza gratuidad en la asistencia y atención de necesidades especiales de niños y adolescentes.

11 Todos los niños reciben instrucciones en su idioma de origen.

12 En caso de adopción, se prioriza lo que es mejor para el bienestar del niño.

13 Se garantiza que los niños no sean ni sometidos a malos tratos o torturas.

14 La supervisión que se brinda a los niños y adolescentes es adecuada.

15 Se prioriza el bienestar del niño ante cualquier decisión que le afecte.

16 Se permite que los niños mantengan contacto regular con ambos padres.

17 Se permite que los niños mantengan contacto directo con ambos padres.

18 El Estado se asegura de que el personal que atiende a niños y adolescentes tenga las competencias necesarias.

19 La detención, encarcelamiento o prisión de un niño se usa como última medida y durante el periodo más breve posible.

20 Se considera inocente al niño acusado de infringir la ley hasta que se pruebe su culpabilidad.

21 Cuando un niño infringe la ley, se le informa directamente los cargos que se le imputan.

22 Cuando se acusa a un niño de haber infringido la ley, se le asiste jurídicamente para su defensa.

23 Se garantiza la supervivencia de los niños.

24 Ante cualquier situación se resguarda el derecho de vida del niño.

25 Se garantiza el mayor desarrollo posible de los niños.

26 Se fomenta que los niños descansen y se recreen considerando su edad.

27 Se asegura atención sanitaria prenatal y posnatal apropiada para madres.

28 Se asegura que todo niño acceda a prestación de asistencia médica.

29 Se disponen alimentos nutritivos adecuados para combatir enfermedades y malnutrición infantil.

30 Se enseña a padres y niños las ventajas de la lactancia materna.

31 Todo niño accede a agua potable salubre.

32 Se desarrolla atención sanitaria preventiva.

33 Se enseña a padres y niños las medidas pertinentes para prevenir accidentes.

34 Se protege a los niños de producción y tráfico ilícito de sustancias.

35 Se asiste a padres para un adecuado desempeño en la crianza y cuidado del niño.

36 Se enseña a padres y niños sobre principios básicos de higiene y saneamiento ambiental.

37 Se promueve la recuperación física y psicológica de niños que han sido víctimas.

38 Se promueve la reintegración social de niños que han sido víctimas.

39 Se asegura la protección y los cuidados necesarios para el bienestar de los niños.

40 La institución cumple con normas establecidas de seguridad para los niños y adolescentes.

41 La institución cumple con las normas de sanidad pertinentes para el trabajo con niños.

42 Se protege a los niños de cualquier forma de explotación y abuso sexual.

43 Se protege al niño contra cualquier forma de abuso.

44 Se protege al niño contra cualquier forma de explotación.

45 Se promueve la integración social de niños con capacidades reducidas.

46 Se garantiza que niños en condición de discapacidad accedan a servicios de rehabilitación.

47 Niños en condición de discapacidad son preparados en lo laboral.
} 


\section{Tabla 2 (continuación)}

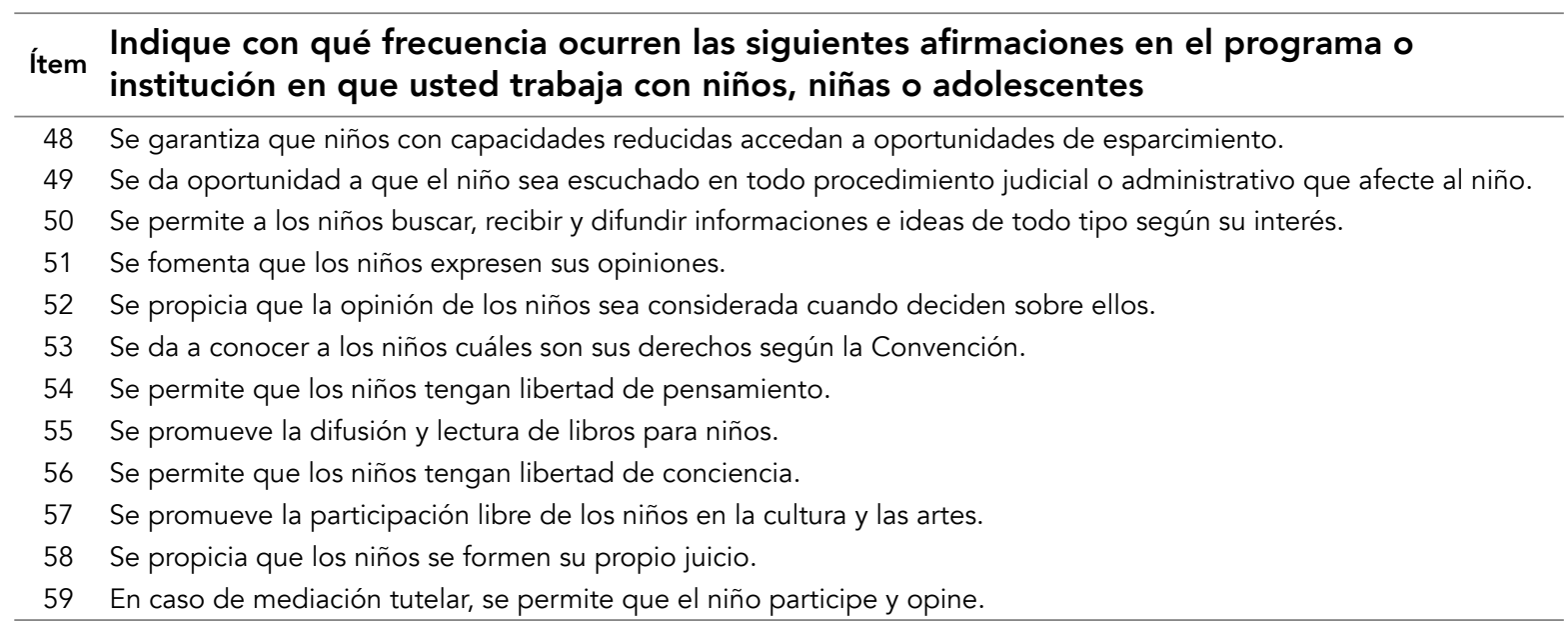

\section{Tabla 3}

Dimensiones e items del instrumento

\begin{tabular}{|c|c|c|c|c|}
\hline & No discriminación & Interés superior & $\begin{array}{l}\text { Derecho a la vida, } \\
\text { desarrollo y } \\
\text { supervivencia }\end{array}$ & Participación \\
\hline $\begin{array}{l}\text { Definición } \\
\text { conceptual }\end{array}$ & $\begin{array}{l}\text { Plantea un trato sin } \\
\text { distinción a los NNA, } \\
\text { independiente de } \\
\text { cualquier condición } \\
\text { propia, de sus proge- } \\
\text { nitores o cuidadores } \\
\text { (Ministerio de Rela- } \\
\text { ciones Exteriores, } \\
\text { 1990). }\end{array}$ & $\begin{array}{l}\text { Implica una mirada } \\
\text { sistémica del NNA y } \\
\text { su contexto. Además } \\
\text { de velar porque todos } \\
\text { sus derechos sean } \\
\text { respetados para ga- } \\
\text { rantizar su desarrollo } \\
\text { integral (Naciones } \\
\text { Unidas, 2013). }\end{array}$ & $\begin{array}{l}\text { Resguarda el derecho } \\
\text { del NNA a vivir y } \\
\text { desarrollarse integral- } \\
\text { mente en un ambiente } \\
\text { propicio para el des- } \\
\text { pliegue de sus poten- } \\
\text { cialidades (Save the } \\
\text { Children, 2009). }\end{array}$ & $\begin{array}{l}\text { Releva la escucha e } \\
\text { intervención de los } \\
\text { NNA en los procesos } \\
\text { de formulación, deci- } \\
\text { sión, implementación } \\
\text { y evaluación de ac- } \\
\text { ciones que les afec- } \\
\text { tan (Giorgi, 2010). }\end{array}$ \\
\hline $\begin{array}{l}\text { Composición } \\
\text { n. }{ }^{\circ} \text { según } \\
\text { tabla } 2\end{array}$ & $\begin{array}{l}10 \text { ítems: 1, 4, 7, 19, } \\
21,27,37,39,48,52 .\end{array}$ & $\begin{array}{l}11 \text { ítems: } 23,28,30 \\
31,45,53,55,56,57 \\
58 \text { y } 59 .\end{array}$ & $\begin{array}{l}28 \text { items: } 2,3,5,6 \\
10,11,12,13,14,16 \\
17,20,22,24,26,32 \\
33,34,35,36,38,40 \\
41,42,46,47,49,54 .\end{array}$ & $\begin{array}{l}10 \text { ítems: } 8,9,15 \\
18,25,29,43,44 \\
50,51 .\end{array}$ \\
\hline
\end{tabular}

\section{Procedimiento}

En la plataforma Lime Survey se creó el instrumento en formato de encuesta en línea, la cual fue enviada a correos electrónicos de operadores sociales, sociedades de profesionales en Chile y grupos de profesionales en redes sociales.

$\mathrm{Al}$ inicio de la encuesta se les invitó a participar si cumplían los criterios de inclusión de la investigación (trabajar o haber trabajado en programas de infancia). Luego, se 
les expuso el consentimiento informado que detallaba el objetivo del estudio, la confidencialidad y voluntariedad de su participación, explicitando la ausencia de compensación material. Además, se indicó su libertad a negarse o suspender su participación en cualquier momento del estudio, sin sufrir daño ni consecuencia alguna por tal acción.

\section{Resultados}

A continuación, se presentan los principales resultados del estudio para cada objetivo. a) Describir la consistencia interna del instrumento a través del alpha de Cronbach: el alpha de Cronbach para la escala completa fue .979 y superior a .87 en cada dimensión (tabla 4 ).

Tabla 4

Alpha de Cronbach

\begin{tabular}{lcccccc}
\hline $\begin{array}{l}\text { Ítems agrupados por } \\
\text { principio (subescalas) }\end{array}$ & Media & Varianza & $\begin{array}{c}\text { Desviación } \\
\text { estándar }\end{array}$ & $\begin{array}{c}\text { n. }{ }^{\circ} \text { de } \\
\text { elementos }\end{array}$ & $\begin{array}{c}\text { n. }{ }^{\circ} \text { de casos } \\
\text { válidos }\end{array}$ & $\begin{array}{c}\text { Alfa de } \\
\text { Cronbach* }\end{array}$ \\
\hline No discriminación & 40.95 & 56.81 & 7.54 & 10 & 230 & .871 \\
Interés superior del niño & 42.54 & 67.99 & 8.246 & 11 & 78 & .887 \\
$\begin{array}{l}\text { Derecho a la vida, } \\
\text { desarrollo y supervivencia }\end{array}$ & 109.29 & 415.94 & 20.39 & 28 & 82 & .957 \\
Participación & 38.87 & 74.84 & 8.65 & 10 & 203 & .930 \\
\hline Escala total & 222.66 & 1970.99 & 44.39 & 59 & 56 & .979 \\
\hline
\end{tabular}

Nota. El análisis consideró 56 casos válidos y eliminación por lista basada en todas las variables del procedimiento. *Basada en elementos estandarizados.

b) Establecer diferencias en la evaluación por dimensión según ámbito de desempeño del programa o institución de los operadores sociales (salud, educación, justicia y desarrollo social) (figura 1). 
Figura 1

Perfiles con grados de cumplimiento de derechos por área de programa

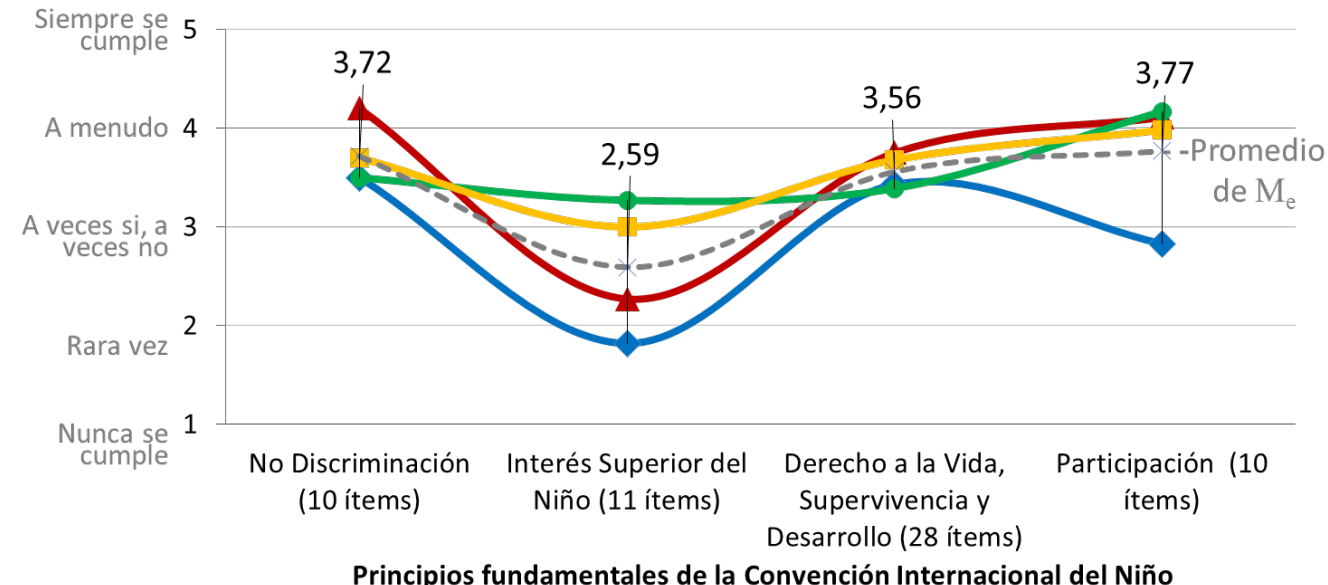

Principios fundamentales de la Convención Internacional del Niño

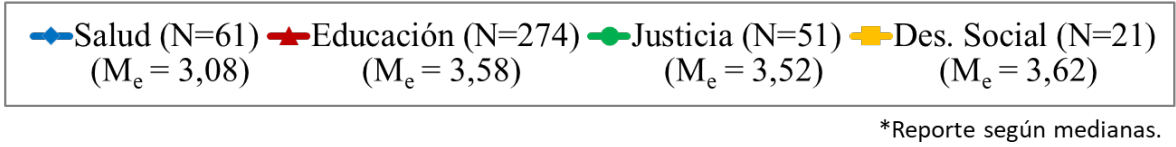

De acuerdo a las medianas reportadas, los operadores sociales en programas e instituciones del área de salud refirieron un menor cumplimiento de derechos vinculados al principio de interés superior, siendo diferencias estadísticamente significativas con las otras tres áreas (educación, justicia y desarrollo social). Así mismo, se observa que las medias de todos los grupos no son iguales con sig. <0.05 (tabla 5).

\section{Tabla 5}

Anova para un factor

\begin{tabular}{llccccc}
\hline $\begin{array}{l}\text { Promedio por principio de la } \\
\text { Convención }\end{array}$ & & $\begin{array}{c}\text { Suma de } \\
\text { cuadrados }\end{array}$ & gl & $\begin{array}{c}\text { Media } \\
\text { cuadrática }\end{array}$ & F & Sig. \\
\hline No discriminación & Inter-grupos & 27.481 & 3 & 9.160 & 14.780 & .000 \\
& Intra-grupos & 249.773 & 403 & .620 & & \\
& Total & 277.254 & 406 & & & \\
Interés superior del Niño & Inter-grupos & 48.924 & 3 & 16.308 & 14.743 & .000 \\
& Intra-grupos & 445.765 & 403 & 1.106 & & \\
Total & 494.688 & 406 & & & \\
Derecho a la vida, desarrollo y & Inter-grupos & 10.763 & 3 & 3.588 & 6.296 & .000 \\
supervivencia & Intra-grupos & 229.654 & 403 & .570 & & \\
& Total & 240.416 & 406 & & & \\
Participación & Inter-grupos & 100.059 & 3 & 33.353 & 32.608 & .000 \\
& Intra-grupos & 412.206 & 403 & 1.023 & & \\
\hline \multirow{2}{*}{ Total } & Total & 512.265 & 406 & & & \\
& Inter-grupos & 20.440 & 3 & 6.813 & 13.895 & .000 \\
& Intra-grupos & 197.604 & 403 & .490 & & \\
\hline
\end{tabular}


Mediante una prueba post hoc (Games-Howell) se observó que la diferencia de medias es significativa al nivel .o5 (tabla 6).

\section{Tabla 6}

Prueba post hoc (Games-Howell)

\begin{tabular}{|c|c|c|c|c|c|c|c|}
\hline \multirow{2}{*}{$\begin{array}{l}\text { Variable de- } \\
\text { pendiente }\end{array}$} & \multirow{2}{*}{$\begin{array}{l}\text { (I) Área } \\
\text { Programa }\end{array}$} & \multirow{2}{*}{$\begin{array}{l}\text { (J) Área } \\
\text { Programa }\end{array}$} & \multirow{2}{*}{$\begin{array}{l}\text { Diferencia } \\
\text { de medias } \\
(\mathrm{I}-\mathrm{J})\end{array}$} & \multirow{2}{*}{ Error típico } & \multirow{2}{*}{ Sig. } & \multicolumn{2}{|c|}{$\begin{array}{c}\text { Intervalo de } \\
\text { confianza al } 95 \%\end{array}$} \\
\hline & & & & & & $\begin{array}{l}\text { Límite } \\
\text { inferior }\end{array}$ & $\begin{array}{l}\text { Límite } \\
\text { superior }\end{array}$ \\
\hline \multirow{4}{*}{ No discriminación } & salud & educación & $-.63534\left(^{\star}\right)$ & .12304 & .000 & -.9581 & -.3125 \\
\hline & educación & salud & $.63534\left(^{\star}\right)$ & .12304 & .000 & .3125 & .9581 \\
\hline & & justicia & $.52216\left(^{\star}\right)$ & .13037 & .001 & .1784 & .8659 \\
\hline & justicia & educación & $-.52216\left(^{\star}\right)$ & .13037 & .001 & -.8659 & -.1784 \\
\hline \multirow{8}{*}{ Interés superior } & salud & educación & $-.47749\left(^{\star}\right)$ & .14875 & .010 & -.8668 & -.0881 \\
\hline & & justicia & $-1.25588(*)$ & .18839 & .000 & -1.7474 & -.7644 \\
\hline & & des. social & $-.97518\left(^{\star}\right)$ & .26307 & .004 & -1.6846 & -.2657 \\
\hline & educación & salud & $.47749\left(^{*}\right)$ & .14875 & .010 & .0881 & .8668 \\
\hline & & justicia & $-.77839\left(^{\star}\right)$ & .14749 & .000 & -1.1658 & -.3909 \\
\hline & justicia & salud & $\left.1.25588^{*}\right)$ & .18839 & .000 & .7644 & 1.7474 \\
\hline & & educación & $\left..778399^{*}\right)$ & .14749 & .000 & .3909 & 1.1658 \\
\hline & des. social & salud & $.97518\left(^{*}\right)$ & .26307 & .004 & .2657 & 1.6846 \\
\hline \multirow{4}{*}{$\begin{array}{l}\text { Derecho a la vida, } \\
\text { desarrollo y super- } \\
\text { vivencia }\end{array}$} & salud & educación & $-.42164\left(^{\star}\right)$ & .13050 & .010 & -.7645 & -.0788 \\
\hline & educación & salud & $.42164\left(^{\star}\right)$ & .13050 & .010 & .0788 & .7645 \\
\hline & & justicia & $.28243(*)$ & .10536 & .044 & .0053 & .5595 \\
\hline & justicia & educación & $-.28243\left(^{\star}\right)$ & .10536 & .044 & -.5595 & -.0053 \\
\hline \multirow{6}{*}{ Participación } & salud & educación & $-1.40095\left(^{\star}\right)$ & .18363 & .000 & -1.8837 & -.9182 \\
\hline & & justicia & $-1.27320\left(^{*}\right)$ & .21654 & .000 & -1.8385 & -.7079 \\
\hline & & des. social & $-1.42961\left(^{\star}\right)$ & .28416 & .000 & -2.1870 & -.6722 \\
\hline & educación & salud & $1.40095\left(^{\star}\right)$ & .18363 & .000 & .9182 & 1.8837 \\
\hline & justicia & salud & $1.27320\left(^{\star}\right)$ & .21654 & .000 & .7079 & 1.8385 \\
\hline & des. social & salud & $\left.1.429611^{(\star}\right)$ & .28416 & .000 & .6722 & 2.1870 \\
\hline
\end{tabular}

Nota. La tabla solo contiene aquellos grupos con diferencia de medias significativa al nivel .o5 $(*)$. 


\section{Discusión}

El presente estudio contribuye en el contexto chileno con la aplicación de un instrumento articulado con los principios fundamentales de la Convención como propuesta para medir los programas y servicios vinculados a la atención infanto-juvenil (Herrera \& Aravena, 2015; Lagos, 2017; Muzzio et al., 2008).

En este sentido, la dimensión de derecho a la vida, desarrollo y supervivencia fue la que estuvo compuesta por más ítems en comparación a las otras tres. Esto podría explicarse por su mayor cantidad de indicadores de evaluación concordantes con la visión de macrodimensiones y estudios existentes sobre condiciones generales de vida desde el siglo XX — cuando se construyó la Convención-y hasta la actualidad (Antilef, 2015; Cepal \& Unicef, 2012).

En términos metodológicos, el tipo de muestreo determinó una conformación disímil entre cada submuestra de participantes por área, lo que dio un resultado preocupante en esta aplicación del instrumento. El $21 \%$ de la muestra (85 participantes) respondió la opción No aplica en 18 a 55 ítems de los 59 del total de la encuesta, razón por la cual la consistencia interna reportada del instrumento debe tomarse con cautela. Aquello puede deberse al riesgo de descontextualización planteado por Magistris (2013) y a las características heterogéneas de la muestra, ya sea por la diversidad de las áreas, programas, instituciones y profesiones que participaron de este estudio, la frecuencia en el contacto con la población atendida (diario en educación vs. esporádico en salud) y los tipos de servicios de atención brindados (prevención universal en desarrollo social vs. tratamiento especializado por vulneración de derechos o por cumplimiento de sanciones penales en justicia).

Un segundo hallazgo importante es que, de todas las variables sociodemográficas de la encuesta, solo área del programa/institución tuvo diferencias estadísticamente significativas entre grupos. Esto hace pensar que hay ítems que responden a la peculiaridad de mandatos técnicos de una institución tal como el ítem 59 («En caso de mediación tutelar, se permite que el niño participe y opine»), vinculado al área de justicia, en comparación a otros ítems que mencionan derechos generales, como el ítem 23 («Se garantiza la supervivencia de los niños»).

Vinculado con lo anterior, resalta que los ítems exclusivos a un área de programa pueden responder a demandas específicas existentes en manuales y protocolos de 
desempeño profesional que delimitan el quehacer de los operadores sociales, lo que pudo facilitar su respuesta y observación en comparación con aquellos tensionados por su postura valórica personal al respecto (Alfaro, 2012; Alfaro \& Zambrano, 2009; Lombaert, 2016).

Como tercer hallazgo, los participantes de la encuesta mencionaron mayor cumplimiento en la dimensión participación, lo cual podría estar relacionado a que los niños y las niñas son escuchados más que antaño, pese a que hay prácticas cotidianas que les invisibilizan o invitan a participar desde un modelo adultocéntrico (Lundy \& Templeton, 2018; Contreras \& Pérez, 2011).

Como cuarto hallazgo, llama la atención que los operadores sociales observaron un menor cumplimiento del interés superior del niño, lo que puede deberse a que nuestra sociedad aún no es capaz de velar por el cumplimiento de este principio o que no se ha asumido activamente el rol de promotores del desarrollo de los niños, niñas y adolescentes como sujetos sociales en formación. Además, ¿será que la definición de la Convención es muy abstracta para quienes tienen la difícil tarea de implementar y cumplir dichos principios? (Rivas, 2015); ¿será que los operadores sociales carecen de formación continua y especializada? (Comité de los Derechos del Niño, 2018); ¿será que el contexto nacional durante la aplicación del instrumento permeó la necesidad de evaluar positivamente todos los derechos para no ser cuestionados profesionalmente?; ¿será que primó la buena imagen institucional al responder según lo demandado por el Programa? (Lombaert, 2016); ¿será que la tendencia a la aquiescencia encubre silenciosamente la inacción de un Estado que naturaliza la vulneración de los derechos humanos? (Jaroslavsky, 2017); ¿será que la falta de conocimiento acerca de todas las normativas vinculadas a los derechos infantiles dificulta que los operadores sociales actúen con un enfoque de derechos en la atención en salud? (Zunino et al., 2018).

Respecto a la evaluación de las cuatro dimensiones realizada por las y los operadores sociales al compararlas con otras encuestas aplicadas a niños, niñas y adolescentes y operadores sociales, se logra ver coherencia sobre el cumplimiento de derechos en la población infantil general (ámbito escolar) que suele mencionar niveles positivos de satisfacción (Programa de las Naciones Unidas para el Desarrollo, 2018; Zunino et al., 2018). Esta evaluación general coexiste con la falta del compromiso y competencia del Estado por aplicar instrumentos especializados en la evaluación de infancia vulnerada (ámbito de justicia), lo que requiere el compromiso mancomunado de los partidos políticos, operadores sociales y la propia infancia (Comité de los Derechos del Niño, 2018; Durán-Strauch, 2017; Herrera \& Aravena, 2015; Naciones Unidas, 2013; Observatorio para la confianza, 2019). 
En concordancia con encuestas nacionales e internacionales con participación de niños, niñas y adolescentes (Corporación Opción, 2010; Programa de las Naciones Unidas para el Desarrollo, 2018; Universidad Central de Chile \& Corporación Opción, 2012), se observa que los operadores sociales del área de educación valoran un mayor cumplimiento en las cuatro dimensiones, pudiendo relacionarse a la consideración del espacio educativo y a los profesores y las profesoras como promotores de difusión y respeto de los derechos de la Convención y Derechos Humanos en general (Gárate et al., 2020). Además, la mejor valoración puede estar relacionada a la mayor cantidad de horas que pasan los niños, niñas y adolescentes en el contexto educativo y a la expectativa social de implicación en sus vidas (Fernández et al., 2018).

En futuras investigaciones, habrá que explorar los hallazgos que no pudieron ser interpretados dada la escasa evidencia empírica. Además, se deberá considerar el enfoque territorial en el instrumento para identificar en qué sectores hay población infantil vulnerada en sus derechos, debiendo intervenir el Estado e instituciones especializadas para protegerla (Oyanedel et al., 2015; Pautassi \& Royo, 2012).

Igualmente, es relevante sistematizar y articular las iniciativas de instrumentos de medición multidimensional de cumplimiento de derechos infantiles, desde la opinión de los niños, niñas y adolescentes, de los/as operadores sociales y cifras oficiales considerando las prácticas cotidianas por tipo de atención y lugar (Tuñón \& González, 2013).

Ante las graves y sistemáticas vulneraciones contra los niños, niñas y adolescentes en Chile queda el desafío de adaptar el presente instrumento para la población infantil como usuaria de servicios y prestaciones (Comité de los Derechos del Niño, 2018; Contreras, 2007; Diálogo Interamericano \& Centro de Estudios Primera Infancia, 2018; Muzzio et al., 2008). De manera particular para la población infantil vulnerada que, comparativamente, tiene más brechas y desigualdades que la población general (Alfaro \& Zambrano, 2009; Naciones Unidas, 2013; Observatorio Niñez y Adolescencia, 2017; Observatorio para la Confianza, 2019; Pereyra, 2020).

También cabe señalar que hay derechos sensibles al quehacer profesional, siendo el personal un posible vulnerador de derechos si no está consciente de su rol promotor, lo que quedó reflejado en el ítem 18 («El Estado se asegura de que el personal que atiende a niños y adolescentes tenga las competencias necesarias») y el ítem 43 («Se protege al niño contra cualquier forma de abuso»). 
Finalmente, desde el aseguramiento de la calidad y la evaluación del impacto de las acciones realizadas en infancia, los resultados de un instrumento adaptado según población y área de ejercicio laboral de operadores sociales, aportaría a contar con «procedimientos eficaces de supervisión del personal que trabaja en el sistema, y su sanción y remoción ante casos de violaciones de los derechos de niños, niñas y adolescentes» (Comité de los Derechos del Niño, 2018, p. 19).

\section{Referencias}

Alfaro, J. (2012). Posibilidades y tensiones en la relación entre psicología comunitaria y políticas sociales. En J. Alfaro, A. Sánchez, \& A. Zambrano (Comps.), Psicología comunitaria y políticas sociales: reflexiones y experiencias (pp. 45-76). Paidós.

Alfaro, J., \& Zambrano, A. (2009). Psicología comunitaria y políticas sociales en Chile. Psicologia \& Sociedade, 21(2), 275-282. https://doi.org/10.159o/So102-71822009000200015

Alkire, S., \& Foster, J. (2011). Counting and multidimensional poverty measurement. Journal of Public Economics, 95(7-8), 476-487. https://doi.org/10.1016/j.jpubeco.2010.11.006

Amador, J. C. (2020). Las voces infantiles llegan a ser tan potentes que es imposible no escucharlas: entrevista a Susana Sosenski. Revista Latinoamericana de Ciencias Sociales, Niñez y Juventud, 18(2), 1-10. https://doi.org/10.1160o/1692715x.18112

Antilef, V. (2015). El gobierno de la pobreza en Chile y en Brasil: consideraciones al concepto de desarrollo social en las políticas e instrumentos de medición [Tesis Doctoral no publicada]. Universidad Academia de Humanismo Cristiano.

Beloff, M. (1990). Modelo de la protección integral de los derechos del niño y de la situación irregular. Justicia y Derechos, (1), 9-21.

Cepal \& Unicef. (2012). Guía para estimar la pobreza infantil. Cepal.

Comité de los Derechos del Niño. (2018). Informe de la investigación relacionada a Chile en virtud del artículo 13 del Protocolo Facultativo de la Convención sobre los Derechos del Niño relativo a un procedimiento de comunicaciones. https://bit.ly/39zagKT

Contreras, C. G., \& Pérez, A. J. (2011). Participación invisible: niñez y prácticas participativas emergentes. Revista Latinoamericana de Ciencias Sociales, Niñez y Juventud, 9(2), 811-825.

Contreras, M. (2007). Pare, mire y actúe: un aporte para incorporar el enfoque de derechos de la niñez en la práctica social. Fundación del Hogar de Cristo y Unicef. https://bit.ly/ $33 \mathrm{GmRIC}$ 
Contreras-Taibo, L., Paulsen-Gutiérrez, C., \& Gómez-Muzzio, E. (2018). Graves vulneraciones de derechos en la infancia y adolescencia: variables de funcionamiento familiar. Universitas Psychologica, 17(3), 1-13. https://doi.org/10.11144/javeriana.upsy17-3.gvdi

Corporación Opción. (2010). El Chile actual y futuro: la visión de los niños, niñas y adolescentes del bicentenario. Autor.

Chzhen, Y., de Neubourg, C., Plavgo, I., \& de Milliano, M. (2016). Child poverty in the European Union: The multiple overlapping deprivation analysis approach (EU-Moda). Child Indicators Research, (9), 335-356. https://doi.org/10.1007/S12187-015-9321-7

Dávila, P., \& Naya, L. (2006). La evolución de los derechos de la infancia: una visión internacional. Encounters in Theory and History of Education, (7), 71-93.

De Neubourg, C., Chai, J., de Milliano, M., \& Plavgo, I. (2012). Cross-country moda study: Multiple Overlapping Deprivation Analysis (Moda). Unicef Office of Research Working Paper.

Diálogo Interamericano \& Centro de Estudios Primera Infancia. (2018). Chile: informe de progreso de políticas de primera infancia. https://bit.ly/2Jqxjxo

Durán-Strauch, E. (2017). Derechos de niños y niñas: del discurso a la política local. Revista Latinoamericana de Ciencias Sociales, Niñez y Juventud, 15(2), 879-891.

Eiroá, F., Fernández, I., \& Pérez, P. (2008). Cuestionarios psicológicos e investigación en internet: una revisión de la literatura. Anales de Psicología, 24(1), $150-157$.

Espíndola, E., Sunkel, G., Murden, A., \& Milosavljevic, V. (2017). Medición multidimensional de la pobreza infantil: una revisión de sus principales componentes teóricos, metodológicos y estadisticos. https://bit.ly/2VrvXUZ

Espinoza-Delgado, J., \& Klasen, S. (2017). Gender and multidimensional poverty in Nicaragua: An individual-based approach. Georg-August-Universität Göttingen; Courant Research Centre-Poverty; Equity and Growth (CRC-PEG).

Fernández, A., González, M. \& Verde, C. (2018). Percepción de profesionales de centros de protección de menores de Galicia sobre la calidad del recurso. Revista Prisma Social, (23), 300-330.

Fondo de las Naciones Unidas para la Infancia. (2015). Definiciones conceptuales para un sistema integral de protección a la infancia. Unicef. https://uni.cf/2VAoFii

Gárate, F., López-Andrada, C., Ocampo-González, A., \& Pincheira, L. (2020). Entrevista a Abraham Magendzo Kolstrein sobre la enseñanza de los Derechos Humanos. Telos: Revista de Estudios Interdisciplinarios en Ciencias Sociales, 22(1), 235-241. https://oi.org/ 10.3639o/telos221.16 
Giorgi, V. (2010). La participación de niños, niñas y adolescentes en las Américas: a 20 años de la Convención sobre los Derechos del Niño. Instituto Interamericano del Niño, la Niña y Adolescentes.

Guio, A.-C., Gordon, D., \& Marlier, E. (2012). Measuring material deprivation in the EU: Indicators for the whole population and child-specific indicators. Office for Official Publications of the European Communities.

Hernández, R., Fernández, C., \& Baptista, P. (2010). Metodología de la investigación. McGraw-Hill.

Herrera, C., \& Aravena, A. (2015). Imaginarios sociales de la infancia en la política social chilena (2001-2012). Revista Latinoamericana de Ciencias Sociales, Niñez y Juventud, $13(1), 72-84$.

Jaroslavsky, L. D. (2017). La aquiescencia y su relación con las fuentes del derecho internacional público: ¿silencio como costumbre? Revista Dat@venia, 9(1), 1-13.

Klasen, S. (2018). Measuring Poverty. Edward Elgar Publishing Limited. https://doi.org/ $10.4337 / 9781786436955$

Lagos, G. (2017). Resultados preliminares de la validación de una encuesta de opinión para evaluar cumplimiento de derechos infantiles en Chile. En S. Sanz (Ed.), El interés superior del niño en la jurisprudencia internacional, comparada y española (pp. 377-388). Aranzadi.

Langford, M., \& Kirkebø, T. (2019). Children's rights' indexes: Measuring Norway's performance. En M. Langford, M. Skivenes, \& K. Harald Søvig (Eds.), Children's Rights in Norway: An Implementation Paradox? (pp. 42-104). Universitetsforlaget. https://doi.org/10.18261/9788215031415-2019-03

Lombaert, E. (2016). La judicialización de conflictos interaccionales que involucran a niños, niñas y adolescentes en las prácticas discursivas de profesionales [Tesis de maestría no publicada]. Universidad de La Frontera.

Lundy, L., \& Templeton, M. (2018). Las opiniones, perspectivas y recomendaciones de niñas, niños y adolescentes alrededor del mundo. Queen's University Belfast; Child Rights Connect. https://bit.ly/2VCyLit

Magistris, G. (2013). El magnetismo de los derechos: desplazamientos y debates en torno a los derechos de niñas, niños y adolescentes. Clacso.

Marina, B. (1998). Las concepciones acerca del «ser niño/a»: el caso de las cuidadoras en instituciones municipales [Tesis de maestría no publicada]. Universidad Académica de Humanismo Cristiano. 
Ministerio de Relaciones Exteriores. (1990). Convención Internacional de los Derechos del Niño en Chile. Biblioteca del Congreso Nacional.

Muzzio, E., Muñoz, M. M., \& Santelices, M. P. (2008). Efectividad de las intervenciones en apego con infancia vulnerada y en riesgo social: un desafío prioritario para Chile. Terapia Psicológica, 26(2), 241-251. https://doi.org/10.4067/s0718-48082008000200010

Naciones Unidas. (2013). Comité de las Naciones Unidas: Observación general n. .14 sobre el derecho del niño a que su interés superior sea una consideración primordial (artículo 3, párrafo 1). Autor.

Observatorio Niñez y Adolescencia. (2017). Cifra negra de violencia sexual contra niñas, niños y adolescentes: ocultamiento social de una tragedia. Reporte I de monitoreo de derechos. https://bit.ly/33XZuur

Observatorio para la Confianza. (2019). Informe 2018: situación de los Derechos Humanos de niños, niñas y adolescentes bajo protección del Estado de Chile. https://bit.ly/2VwubCf

Observatorio Social. (2015). Nueva metodología de medición de la pobreza por ingresos y multidimensional. Ministerio de Desarrollo Social. https://bit.ly/2VwBYzJ

Oyanedel, J. C., Alfaro, J., \& Mella, C. (2015). Bienestar subjetivo y calidad de vida en la infancia en Chile. Revista Latinoamericana de Ciencias Sociales, Niñez y Juventud, 13(1), 313-327.

Pautassi, L. C., \& Royo, L. (2012). Enfoque de derechos en las políticas de infancia: indicadores para su medición. Proyecto Cepal-Unicef UNI/12/oo1. Naciones Unidas.

Pereyra, E. J. (2020). Proyectos de gobierno y desarrollo de institucionalidad juvenil: intensidades y tensiones del vínculo en Argentina (1989-2015). Revista Latinoamericana de Ciencias Sociales, Niñez y Juventud, 18(1), 1-31. https://doi.org/10.1160o/1692715x.18108

Programa de las Naciones Unidas para el Desarrollo. (2018). Primera encuesta de desarrollo humano en niños, niñas y adolescentes. https://bit.ly/3fUHBRV

Rivas, E. (2015). La evolución del interés superior del niño: hacia una evaluación y determinación objetiva. Universidad de Chile.

Save the Children. (2009). Convención sobre los Derechos del Niño: Programa de Promoción y Defensa de los Derechos de la Infancia. Autor.

Sen, A. K. (1979). Issues in the measurement of poverty. The Scandinavian Journal of Economics, 81(2), 285-307.

Sola-Morales, S., \& Campos-Garrido, N. A. (2019). Discurso estatal chileno en la protección de los derechos de niños(as) y jóvenes. Revista Latinoamericana de Ciencias Sociales, Niñez y Juventud, 17(1), 105-124. https://doi.org/10.1160o/1692715x.17106 
Thorbecke, E., (2008). Multidimensional poverty: Conceptual and measurement issues, En N. Kakwani, \& J. Silber (Eds.), The many dimensions of poverty (pp. 89-119). Palgrave Macmillan. https://doi.org/10.1057/9780230592407_1

Tunón, I., \& González, M. S. (2013). Aproximación a la medición de la pobreza infantil desde un enfoque multidimensional y de derechos. Revista Sociedad \& Equidad, (5), 30-6o. https://doi.org/10.5354/0718-9990.2013.26317

Universidad Central de Chile \& Corporación Opción. (2012). Niños, niñas y derechos humanos: nuevos actores y nuevas visiones: principales resultados de la Encuesta nacional de derechos humanos y niños, niñas y adolescentes. LOM.

Zunino, C., Sánchez, V., Romero, M., \& Giachetto, G. (2018). Encuesta a posgrados de pediatría sobre conocimiento y aplicación de los derechos de los niños y adolescentes en la práctica clínica. Anales de la Facultad de Medicina de la Universidad de La República, 5(1), 10-17. https://doi.org/10.25184/anfamed2018v5n1a2 